\title{
Minimal Wave Speed in a Predator-Prey System with Distributed Time Delay
}

\author{
Fuzhen Wu (i) and Dongfeng Li \\ Department of Basic, Zhejiang University of Water Resources and Electric Power, Hangzhou, Zhejiang 310018, China \\ Correspondence should be addressed to Fuzhen Wu; fuzhwu@yeah.net
}

Received 14 March 2018; Accepted 6 May 2018; Published 31 May 2018

Academic Editor: Nikos I. Karachalios

Copyright (C) 2018 Fuzhen Wu and Dongfeng Li. This is an open access article distributed under the Creative Commons Attribution License, which permits unrestricted use, distribution, and reproduction in any medium, provided the original work is properly cited.

\begin{abstract}
This paper is concerned with the minimal wave speed of traveling wave solutions in a predator-prey system with distributed time delay, which does not satisfy comparison principle due to delayed intraspecific terms. By constructing upper and lower solutions, we obtain the existence of traveling wave solutions when the wave speed is the minimal wave speed. Our results complete the known conclusions and show the precisely asymptotic behavior of traveling wave solutions.
\end{abstract}

\section{Introduction}

Traveling wave solutions of predator-prey systems have been widely utilized to model population invasion, and the minimal wave speed of traveling wave solutions is often regarded as an important threshold to characterize the invasion feature in many examples, see Owen and Lewis [1] and Shigesada and Kawasaki [2, Chapter 8]. Moreover, Lin [3] and Pan [4] confirmed that, in a Lotka-Volterra type system, the minimal wave speed of invasion traveling wave solutions is equal to the invasion speed of the predator. Here, the invasion speed is estimated by the corresponding initial value problem when the initial value of the predator admits a nonempty compact support.

When the wave system of predator-prey system is of finite dimension, there are many important results, for example, the earlier results by Dunbar [5-7]. But when the corresponding wave system is of infinite dimension, there are some open problems on the minimal wave speed, for example, in the following system [8]:

$$
\begin{aligned}
& \frac{\partial u_{1}(x, t)}{\partial t}=d_{1} \Delta u_{1}(x, t)+r_{1} u_{1}(x, t) F_{1}\left(u_{1}, u_{2}\right)(x, t), \\
& \frac{\partial u_{2}(x, t)}{\partial t}=d_{2} \Delta u_{2}(x, t)+r_{2} u_{2}(x, t) F_{2}\left(u_{1}, u_{2}\right)(x, t),
\end{aligned}
$$

where $x \in \mathbb{R}, t>0, d_{1}>0, d_{2}>0, r_{1}>0, r_{2}>0, u_{1} \in \mathbb{R}$, $u_{2} \in \mathbb{R}$, and

$$
\begin{aligned}
F_{1}\left(u_{1}, u_{2}\right)(x, t)= & 1-u_{1}(x, t) \\
& -a_{1} \int_{-\tau}^{0} u_{1}(x, t+s) d \eta_{11}(s) \\
& -b_{1} \int_{-\tau}^{0} u_{2}(x, t+s) d \eta_{12}(s), \\
F_{2}\left(u_{1}, u_{2}\right)(x, t)= & 1-u_{2}(x, t) \\
& -a_{2} \int_{-\tau}^{0} u_{2}(x, t+s) d \eta_{22}(s) \\
& +b_{2} \int_{-\tau}^{0} u_{1}(x, t+s) d \eta_{21}(s),
\end{aligned}
$$

in which $a_{1} \geq 0, a_{2} \geq 0, b_{1} \geq 0, b_{2} \geq 0$, and $\tau \geq 0$ are constants such that

$\eta_{i j}(s)$ is nondecreasing on $[-\tau, 0]$,

$$
\eta_{i j}(0)-\eta_{i j}(-\tau)=1,
$$

$$
i, j=1,2 \text {. }
$$


For (1), a traveling wave solution is a special solution with the form

$$
u_{i}(x, t)=\phi_{i}(\xi), \quad \xi=x+c t, i=1,2,
$$

where $\left(\phi_{1}, \phi_{2}\right) \in C^{2}\left(\mathbb{R}, \mathbb{R}^{2}\right)$ is the wave profile and $c>0$ is the wave speed. Therefore, $\left(\phi_{1}, \phi_{2}\right)$ and $c$ satisfy

$$
\begin{array}{ll}
d_{1} \phi_{1}^{\prime \prime}(\xi)-c \phi_{1}^{\prime}(\xi)+r_{1} \phi_{1}(\xi) F_{1}\left(\phi_{1}, \phi_{2}\right)(\xi)=0, & \\
\xi \in \mathbb{R}, & \\
d_{2} \phi_{2}^{\prime \prime}(\xi)-c \phi_{2}^{\prime}(\xi)+r_{2} \phi_{2}(\xi) F_{2}\left(\phi_{1}, \phi_{2}\right)(\xi)=0, & \\
\xi \in \mathbb{R}
\end{array}
$$

with

$$
\begin{aligned}
F_{1}\left(\phi_{1}, \phi_{2}\right)(\xi)= & 1-\phi_{1}(\xi)-a_{1} \int_{-\tau}^{0} \phi_{1}(\xi+c s) d \eta_{11}(s) \\
& -b_{1} \int_{-\tau}^{0} \phi_{2}(\xi+c s) d \eta_{12}(s), \\
F_{2}\left(\phi_{1}, \phi_{2}\right)(\xi)= & 1-\phi_{2}(\xi)-a_{2} \int_{-\tau}^{0} \phi_{2}(\xi+c s) d \eta_{22}(s) \\
& +b_{2} \int_{-\tau}^{0} \phi_{1}(\xi+c s) d \eta_{21}(s) .
\end{aligned}
$$

In Pan [8], the author defined a threshold given by

$$
c^{*}:=\max \left\{2 \sqrt{d_{1} r_{1}}, 2 \sqrt{d_{2} r_{2}}\right\}
$$

and showed the existence (nonexistence) of desired traveling wave solutions if the wave speed $c>c^{*}\left(c<c^{*}\right)$. When the wave speed $c=c^{*}$, the author presented the existence of traveling wave solutions under special conditions. Besides [8], there are also some results on the existence of traveling wave solutions of predator-prey models similar to (1) when the wave speed is large; see Huang and Zou [9], K. Li and X. Li [10], and Lin et al. [11].

The purpose of this paper is to confirm the existence of nontrivial traveling wave solutions of (1) without other conditions when the wave speed $c=c^{*}$. Since Pan $[8$, Theorem 3.5] also holds when $c=c^{*}$, we shall not investigate the limit behavior as $\xi \rightarrow \infty$ and focus on the existence of positive solution of (5) satisfying

$$
\lim _{\xi \rightarrow-\infty} \phi_{i}(\xi)=0, \quad i=1,2
$$

Motivated by Lin and Ruan [12] on an abstract result of traveling wave solutions of delayed reaction-diffusion systems, we shall construct proper upper and lower solutions similar to those in Fu [13] and Lin [14] to study the existence of traveling wave solutions.

\section{Main Results}

When $c=c^{*}$, we define

$$
\begin{aligned}
& \gamma_{1}=\frac{c-\sqrt{c^{2}-4 d_{1} r_{1}}}{2 d_{1}} \\
& \gamma_{2}=\frac{c-\sqrt{c^{2}-4 d_{2} r_{2}}}{2 d_{2}} .
\end{aligned}
$$

By these constants, we first present our main conclusion as follows.

Theorem 1. Assume that $c=c^{*}$ holds. Then (5) admits a bounded positive solution $\left(\phi_{1}, \phi_{2}\right)$ satisfying

(1) $\lim _{\xi \rightarrow-\infty}\left(\phi_{1}(\xi) /-\xi e^{\gamma_{1} \xi}\right)=1, \lim _{\xi \rightarrow-\infty}\left(\phi_{2}(\xi) / e^{\gamma_{2} \xi}\right)=1$ if $c=c^{*}=2 \sqrt{d_{1} r_{1}}>2 \sqrt{d_{2} r_{2}}$;

(2) $\lim _{\xi \rightarrow-\infty}\left(\phi_{1}(\xi) / e^{\gamma_{1} \xi}\right)=1, \lim _{\xi \rightarrow-\infty}\left(\phi_{2}(\xi) /-\xi e^{\gamma_{2} \xi}\right)=1$ if $c=c^{*}=2 \sqrt{d_{2} r_{2}}>2 \sqrt{d_{1} r_{1}}$;

(3) $\lim _{\xi \rightarrow-\infty}\left(\phi_{1}(\xi) /-\xi e^{\gamma_{1} \xi}\right)=1, \lim _{\xi \rightarrow-\infty}\left(\phi_{2}(\xi) /-\xi e^{\gamma_{2} \xi}\right)=$ 1 if $c=c^{*}=2 \sqrt{d_{2} r_{2}}=2 \sqrt{d_{1} r_{1}}$.

We shall prove the result by three lemmas, which will study three cases $d_{1} r_{1}>d_{2} r_{2}, d_{1} r_{1}<d_{2} r_{2}$, and $d_{1} r_{1}=d_{2} r_{2}$. For this purpose, we first show the following result in Lin and Ruan [12].

Lemma 2. Suppose that $\underline{\phi}_{1}(\xi), \bar{\phi}_{1}(\xi), \underline{\phi}_{2}(\xi)$, and $\bar{\phi}_{2}(\xi)$ are continuous functions and

(A1) $0 \leq \underline{\phi}_{1}(\xi) \leq \bar{\phi}_{1}(\xi) \leq 1,0 \leq \underline{\phi}_{2}(\xi) \leq \bar{\phi}_{2}(\xi) \leq 1+b_{2}$, $\xi \in \mathbb{R} ;$

(A2) they are twice differentiable except a set E containing finite points of $\mathbb{R}$ and

$\underline{\phi}_{1}^{\prime}(\xi), \bar{\phi}_{1}^{\prime}(\xi), \underline{\phi}_{2}^{\prime}(\xi), \bar{\phi}_{2}^{\prime}(\xi), \underline{\phi}_{1}^{\prime \prime}(\xi), \bar{\phi}_{1}^{\prime \prime}(\xi), \underline{\phi}_{2}^{\prime \prime}(\xi), \bar{\phi}_{2}^{\prime \prime}(\xi)$

are continuous and bounded if $\xi \in \mathbb{R} \backslash E$;

(A3) when $x \in E$, they satisfy

$$
\begin{aligned}
& \underline{\phi}_{1}^{\prime}(\xi-) \leq \underline{\phi}_{1}^{\prime}(\xi+), \\
& \underline{\phi}_{2}^{\prime}(\xi-) \leq \underline{\phi}_{2}^{\prime}(\xi+), \\
& \bar{\phi}_{1}^{\prime}(\xi+) \leq \bar{\phi}_{1}^{\prime}(\xi-), \\
& \bar{\phi}_{2}^{\prime}(\xi+) \leq \bar{\phi}_{2}^{\prime}(\xi-) ;
\end{aligned}
$$


(A4) they satisfy the following inequalities:

$$
\begin{aligned}
& d_{1} \bar{\phi}_{1}^{\prime \prime}(\xi)-c \bar{\phi}_{1}^{\prime}(\xi)+r_{1} \bar{\phi}_{1}(\xi)\left[1-\bar{\phi}_{1}(\xi)\right. \\
& -a_{1} \int_{-\tau}^{0} \underline{\phi}_{1}(\xi+c s) d \eta_{11}(s) \\
& \left.-b_{1} \int_{-\tau}^{0} \underline{\phi}_{2}(\xi+c s) d \eta_{12}(s)\right] \leq 0 \text {, } \\
& d_{2} \bar{\phi}_{2}^{\prime \prime}(\xi)-c \bar{\phi}_{2}^{\prime}(\xi)+r_{2} \bar{\phi}_{2}(\xi)\left[1-\bar{\phi}_{2}(\xi)\right. \\
& -a_{2} \int_{-\tau}^{0} \underline{\phi}_{2}(\xi+c s) d \eta_{22}(s) \\
& \left.+b_{2} \int_{-\tau}^{0} \bar{\phi}_{1}(\xi+c s) d \eta_{21}(s)\right] \leq 0 \\
& d_{1} \underline{\phi}_{1}^{\prime \prime}(\xi)-c \underline{\phi}_{1}^{\prime}(\xi)+r_{1} \underline{\phi}_{1}(\xi)\left[1-\underline{\phi}_{1}(\xi)\right. \\
& -a_{1} \int_{-\tau}^{0} \bar{\phi}_{1}(\xi+c s) d \eta_{11}(s) \\
& \left.-b_{1} \int_{-\tau}^{0} \bar{\phi}_{2}(\xi+c s) d \eta_{12}(s)\right] \geq 0, \\
& d_{2} \underline{\phi}_{2}^{\prime \prime}(\xi)-c \underline{\phi}_{2}^{\prime}(\xi)+r_{2} \underline{\phi}_{2}(\xi)\left[1-\underline{\phi}_{2}(\xi)\right. \\
& -a_{2} \int_{-\tau}^{0} \bar{\phi}_{2}(\xi+c s) d \eta_{22}(s) \\
& \left.+b_{2} \int_{-\tau}^{0} \underline{\phi}_{1}(\xi+c s) d \eta_{21}(s)\right] \geq 0
\end{aligned}
$$

$$
\text { for } \xi \in \mathbb{R} \backslash E \text {. }
$$

Then (5) has a positive solution $\left(\phi_{1}(\xi), \phi_{2}(\xi)\right)$ such that

$$
\begin{aligned}
& \underline{\phi}_{1}(\xi) \leq \phi_{1}(\xi) \leq \bar{\phi}_{1}(\xi), \\
& \underline{\phi}_{2}(\xi) \leq \phi_{2}(\xi) \leq \bar{\phi}_{2}(\xi),
\end{aligned}
$$

\section{$\xi \in \mathbb{R}$}

Remark 3. In the above lemma, $\left(\bar{\phi}_{1}(\xi), \bar{\phi}_{2}(\xi)\right)$ and $\left(\phi_{1}(\xi), \phi_{2}(\xi)\right)$ are a pair of (generalized) upper and lower solutions of (5). That is, the existence of positive solutions of (5) can be obtained by the existence of (generalized) upper and lower solutions of (5).

Lemma 4. Assume that $d_{1} r_{1}>d_{2} r_{2}$. Then (1) of Theorem 1 holds.

Proof. For simplicity, we shall denote $c^{*}=2 \sqrt{d_{1} r_{1}}$ by $c$ and define

$$
\gamma_{3}=\frac{c+\sqrt{c^{2}-4 d_{2} r_{2}}}{2 d_{2}} .
$$

Let $K>0$ be a constant such that

(K1) $(-\xi+K) e^{\gamma_{1} \xi}$ is monotone if $\xi \leq 0$;

(K2) $K>1$ or $\sup _{\xi \leq 0}(-\xi+K) e^{\gamma_{1} \xi}>1$.

Moreover, select $\eta>1$ with

$$
\eta \gamma_{2}<\min \left\{\gamma_{2}+\frac{\gamma_{1}}{2}, 2 \gamma_{2}, \gamma_{3}\right\}
$$

and $M>1+b_{2}$ such that

$$
\begin{aligned}
& \text { (M1) } e^{\gamma_{2} \xi}+M e^{\eta \gamma_{2} \xi}<1+b_{2} \text { implies }(-\xi+K) e^{\gamma_{1} \xi} \leq e^{\gamma_{1} \xi / 2} ; \\
& \text { (M2) } M>-2 r_{2} b_{2} /\left(d_{2} \eta^{2} \gamma_{2}^{2}-c \eta \gamma_{2}+r_{2}\right)+1+b_{2} ; \\
& \text { (M3) } e^{\gamma_{2} \xi}+M e^{\eta \gamma_{2} \xi}<1+b_{2} \text { implies }\left(d_{2} \eta^{2} \gamma_{2}^{2}-c \eta \gamma_{2}+r_{2}\right)+ \\
& \quad 2 r_{2} b_{2} e^{\gamma_{1} \xi / 2}<0,
\end{aligned}
$$

and $N>1$ such that

(N1) $e^{\gamma_{2} \xi}-N e^{\eta \gamma_{2} \xi}>0$ implies $e^{\gamma_{2} \xi}+M e^{\eta \gamma_{2} \xi}<2 e^{\gamma_{2} \xi}$

(N2) $N>-4 r_{2}\left(1+a_{2}\right) /\left(d_{2} \eta^{2} \gamma_{2}^{2}-c \eta \gamma_{2}+r_{2}\right)+1$.

Select $L>1$ such that

(L1) $\xi<-L^{2}$ implies $(-\xi+K) e^{\gamma_{1} \xi}<e^{\gamma_{1}^{\prime} \xi}<1$, where $\gamma_{1}^{\prime} \epsilon$ $\left(0, \gamma_{1}\right)$ such that

$$
\begin{aligned}
2 \gamma_{1}^{\prime}-\gamma_{1} & >0, \\
\gamma_{1}^{\prime}+\gamma_{2}-\gamma_{1} & >0 ;
\end{aligned}
$$

(L2) $\xi<-L^{2}$ implies $e^{\gamma_{2} \xi}+M e^{\eta \gamma_{2} \xi}<2 e^{\gamma_{2} \xi}$;

(L3) $L>\sup _{\xi<-1}\left(4 \sqrt{(-\xi)^{3}}\left[r_{1}\left(1+a_{1}\right) e^{\left(2 \gamma_{1}^{\prime}-\gamma_{1}\right) \xi}+\right.\right.$ $\left.\left.2 r_{1} b_{1} e^{\left(\gamma_{1}^{\prime}+\gamma_{2}-\gamma_{1}\right) \xi}\right] / d_{1}\right)$.

The admissibility of $L, M, N$, and $K$ is clear by the limit behavior of these functions as $\xi \rightarrow-\infty$. Mathematically, we first fix $K$, then select $M$, and finally define $N, L$. Here, $N$ and $L$ are independent of each other.

We now define

$$
\begin{aligned}
& \bar{\phi}_{1}(\xi)= \begin{cases}(-\xi+K) e^{\gamma_{1} \xi}, & \xi \leq \xi_{1}, \\
1, & \xi \geq \xi_{1},\end{cases} \\
& \underline{\phi}_{1}(\xi)= \begin{cases}(-\xi-L \sqrt{-\xi}) e^{\gamma_{1} \xi}, & \xi \leq-L^{2}, \\
0, & \xi>-L^{2},\end{cases}
\end{aligned}
$$

where $\xi_{1}<0$ such that $\bar{\phi}_{1}(\xi)$ is continuous by (K1)-(K2) and

$$
\begin{aligned}
& \bar{\phi}_{2}(\xi)=\min \left\{e^{\gamma_{2} \xi}+M e^{\eta \gamma_{2} \xi}, 1+b_{2}\right\}, \\
& \underline{\phi}_{2}(\xi)=\max \left\{0, e^{\gamma_{2} \xi}-N e^{\eta \gamma_{2} \xi}\right\} .
\end{aligned}
$$

If these functions satisfy (12), then our result holds by Lemma 2. Now, we are in a position of verifying these 
inequalities. For $\bar{\phi}_{1}(\xi)$, we shall prove the first inequality of (12) when $\xi \neq \xi_{1}$. If $\xi>\xi_{1}$ and $\bar{\phi}_{1}(\xi)=1$, then

$$
\begin{array}{r}
1-\bar{\phi}_{1}(\xi)-a_{1} \int_{-\tau}^{0} \underline{\phi}_{1}(\xi+c s) d \eta_{11}(s) \\
-b_{1} \int_{-\tau}^{0} \underline{\phi}_{2}(\xi+c s) d \eta_{12}(s) \leq 0
\end{array}
$$

and the first inequality of (12) is clear. When $\bar{\phi}_{1}(\xi)=(-\xi+$ $K) e^{\gamma_{1} \xi}<1$, then

$$
\begin{aligned}
d_{1} \bar{\phi}_{1}^{\prime \prime}(\xi)-c \bar{\phi}_{1}^{\prime}(\xi)+r_{1} \bar{\phi}_{1}(\xi) \\
=(-\xi+K)\left(d_{1} \gamma_{1}^{2}-c \gamma_{1}+r_{1}\right) e^{\gamma_{1} \xi} \\
\quad-\left(2 d_{1} \gamma_{1}-c\right) e^{\gamma_{1} \xi}=0
\end{aligned}
$$

and the verification on the first inequality of (12) is finished.

When the second inequality on $\bar{\phi}_{2}(\xi)$ is concerned, it is also clear if $\bar{\phi}_{2}(\xi)=1+b_{2}<e^{\gamma_{2} \xi}+M e^{\eta \gamma_{2} \xi}$. When $\bar{\phi}_{2}(\xi)=$ $e^{\gamma_{2} \xi}+M e^{\eta \gamma_{2} \xi}<1+b_{2}$, then (M1) leads to

$$
\begin{aligned}
& r_{2} \bar{\phi}_{2}(\xi)\left[1-\bar{\phi}_{2}(\xi)-a_{2} \int_{-\tau}^{0} \underline{\phi}_{2}(\xi+c s) d \eta_{22}(s)\right. \\
& \left.\quad+b_{2} \int_{-\tau}^{0} \bar{\phi}_{1}(\xi+c s) d \eta_{21}(s)\right] \leq r_{2} \bar{\phi}_{2}(\xi)[1 \\
& \left.\quad+b_{2} \bar{\phi}_{1}(\xi)\right] \leq r_{2}\left(e^{\gamma_{2} \xi}+M e^{\eta \gamma_{2} \xi}\right)\left(1+b_{2} e^{\gamma_{1} \xi / 2}\right) \\
& \quad=r_{2}\left(e^{\gamma_{2} \xi}+M e^{\eta \gamma_{2} \xi}\right)+r_{2} b_{2}\left(e^{\left(\gamma_{2}+\gamma_{1} / 2\right) \xi}\right. \\
& \left.\quad+M e^{\left(\eta \gamma_{2}+\gamma_{1} / 2\right) \xi}\right) .
\end{aligned}
$$

Note that

$$
\begin{aligned}
d_{2} \bar{\phi}_{2}^{\prime \prime}(\xi)-c \bar{\phi}_{2}^{\prime}(\xi)= & \left(d_{2} \gamma_{2}^{2}-c \gamma_{2}\right) e^{\gamma_{2} \xi} \\
& +M\left(d_{2} \eta^{2} \gamma_{2}^{2}-c \eta \gamma_{2}\right) e^{\eta \gamma_{2} \xi}
\end{aligned}
$$

then the definition of $\gamma_{2}$ implies that the desired inequality is true if

$$
\begin{aligned}
& M\left(d_{2} \eta^{2} \gamma_{2}^{2}-c \eta \gamma_{2}+r_{2}\right) e^{\eta \gamma_{2} \xi} \\
& \quad+r_{2} b_{2}\left(e^{\left(\gamma_{2}+\gamma_{1} / 2\right) \xi}+M e^{\left(\eta \gamma_{2}+\gamma_{1} / 2\right) \xi}\right) \leq 0
\end{aligned}
$$

or

$$
\begin{aligned}
2 M & \left(d_{2} \eta^{2} \gamma_{2}^{2}-c \eta \gamma_{2}+r_{2}\right) \\
& +2 r_{2} b_{2}\left(e^{\left(\gamma_{2}+\gamma_{1} / 2-\eta \gamma_{2}\right) \xi}+M e^{\gamma_{1} \xi / 2}\right) \leq 0 .
\end{aligned}
$$

On the one hand, (M2) leads to

$$
M\left(d_{2} \eta^{2} \gamma_{2}^{2}-c \eta \gamma_{2}+r_{2}\right)+2 r_{2} b_{2}<0
$$

At the same time, we have

$$
\left(d_{2} \eta^{2} \gamma_{2}^{2}-c \eta \gamma_{2}+r_{2}\right)+2 r_{2} b_{2} e^{\gamma_{1} \xi / 2}<0
$$

by (M3). Therefore, (23) is true, as is the case for the second inequality of (12).

On the third inequality of (12), it is clear if $\underline{\phi}_{1}(\xi)=0$. Otherwise,

$$
\begin{gathered}
\underline{\phi}_{1}^{\prime}(\xi)=\gamma_{1} \underline{\phi}_{1}(\xi)-e^{\gamma_{1} \xi}+\frac{L}{2 \sqrt{-\xi}} e^{\gamma_{1} \xi} \\
\underline{\phi}_{1}^{\prime \prime}(\xi)=\gamma_{1}^{2} \underline{\phi}_{1}(\xi)-2 \gamma_{1} e^{\gamma_{1} \xi}+\frac{L \gamma_{1}}{\sqrt{-\xi}} e^{\gamma_{1} \xi} \\
+\frac{L}{4 \sqrt{(-\xi)^{3}}} e^{\gamma_{1} \xi}
\end{gathered}
$$

With these results, we obtain

$$
\begin{aligned}
& d_{1} \underline{\phi}_{1}^{\prime \prime}(\xi)-c \underline{\phi}_{1}^{\prime}(\xi)+r_{1} \underline{\phi}_{1}(\xi)=\left(d_{1} \gamma_{1}^{2}-c \gamma_{1}+r_{1}\right) \\
& \cdot \underline{\phi}_{1}(\xi)-\left(2 d_{1} \gamma_{1}-c\right) e^{\gamma_{1} \xi}+L e^{\gamma_{1} \xi}\left[\frac{d_{1} \gamma_{1}}{\sqrt{-\xi}}-\frac{c}{2 \sqrt{-\xi}}\right. \\
& \left.+\frac{d_{1}}{4 \sqrt{(-\xi)^{3}}}\right]=\frac{L d_{1} e^{\gamma_{1} \xi}}{4 \sqrt{(-\xi)^{3}}}, \\
& r_{1} \underline{\phi}_{1}(\xi)\left[-\underline{\phi}_{1}(\xi)-a_{1} \int_{-\tau}^{0} \bar{\phi}_{1}(\xi+c s) d \eta_{11}(s)\right. \\
& \left.-b_{1} \int_{-\tau}^{0} \bar{\phi}_{2}(\xi+c s) d \eta_{12}(s)\right] \geq-r_{1} \underline{\phi}_{1}^{2}(\xi) \\
& -r_{1} a_{1} \bar{\phi}_{1}(\xi) \int_{-\tau}^{0} \bar{\phi}_{1}(\xi+c s) d \eta_{11}(s)-r_{1} b_{1} \bar{\phi}_{1}(\xi) \\
& \cdot \int_{-\tau}^{0} \bar{\phi}_{2}(\xi+c s) d \eta_{12}(s) \geq-r_{1}\left(1+a_{1}\right) \\
& \cdot \bar{\phi}_{1}^{2}(\xi)-2 r_{1} b_{1} \bar{\phi}_{1}(\xi) \bar{\phi}_{2}(\xi) \geq-r_{1}\left(1+a_{1}\right) e^{2 \gamma_{1}^{\prime} \xi} \\
& -2 r_{1} b_{1} e^{\left(\gamma_{1}^{\prime}+\gamma_{2}\right) \xi}
\end{aligned}
$$

by (L1)-(L2). Therefore, it suffices to prove that

$$
\frac{L d_{1} e^{\gamma_{1} \xi}}{4 \sqrt{(-\xi)^{3}}} \geq r_{1}\left(1+a_{1}\right) e^{2 \gamma_{1}^{\prime} \xi}+2 r_{1} b_{1} e^{\left(\gamma_{1}^{\prime}+\gamma_{2}\right) \xi}
$$

or

$$
\frac{L d_{1}}{4 \sqrt{(-\xi)^{3}}} \geq r_{1}\left(1+a_{1}\right) e^{\left(2 \gamma_{1}^{\prime}-\gamma_{1}\right) \xi}+2 r_{1} b_{1} e^{\left(\gamma_{1}^{\prime}+\gamma_{2}-\gamma_{1}\right) \xi},
$$

which is true by (L3). 
We now consider $\underline{\phi}_{2}(\xi)$, that is, the forth inequality of (12). When $\underline{\phi}_{2}(\xi)>0$, the definition implies

$$
\begin{aligned}
r_{2} & {\left[-\underline{\phi}_{2}^{2}(\xi)-a_{2} \underline{\phi}_{2}(\xi) \int_{-\tau}^{0} \bar{\phi}_{2}(\xi+c s) d \eta_{22}(s)\right.} \\
& \left.+b_{2} \underline{\phi}_{2}(\xi) \int_{-\tau}^{0} \underline{\phi}_{1}(\xi+c s) d \eta_{21}(s)\right] \\
& \geq r_{2}\left[-\underline{\phi}_{2}^{2}(\xi)-a_{2} \underline{\phi}_{2}(\xi) \int_{-\tau}^{0} \bar{\phi}_{2}(\xi+c s) d \eta_{22}(s)\right] \\
& \geq r_{2}\left[-\bar{\phi}_{2}^{2}(\xi)-a_{2} \bar{\phi}_{2}(\xi) \int_{-\tau}^{0} \bar{\phi}_{2}(\xi+c s) d \eta_{22}(s)\right] \\
& \geq-4 r_{2}\left(1+a_{2}\right) e^{2 \gamma_{2} \xi}
\end{aligned}
$$

by (N1) as well as

$$
\begin{aligned}
d_{2} \underline{\phi}_{2}^{\prime \prime}(\xi)-c \underline{\phi}_{2}^{\prime}(\xi)+r_{2} \underline{\phi}_{2}(\xi) \\
=e^{\gamma_{2} \xi}\left(d_{2} \gamma_{2}^{2}-c \gamma_{2}+r_{2}\right) \\
\quad-N e^{\eta \gamma_{2} \xi}\left(d_{2} \eta^{2} \gamma_{2}^{2}-c \eta \gamma_{2}+r_{2}\right) \\
=-N e^{\eta \gamma_{2} \xi}\left(d_{2} \eta^{2} \gamma_{2}^{2}-c \eta \gamma_{2}+r_{2}\right) .
\end{aligned}
$$

Thus, the desired inequality is true if

$$
N>\frac{-4 r_{2}\left(1+a_{2}\right)}{d_{2} \eta^{2} \gamma_{2}^{2}-c \eta \gamma_{2}+r_{2}}+1>1
$$

since $\xi<0$ such that $e^{2 \gamma_{2} \xi}<e^{\eta \gamma_{2} \xi}$, which holds by (N2). The proof is complete.

Lemma 5. Assume that $d_{1} r_{1}<d_{2} r_{2}$. Then (2) of Theorem 1 is true.

Proof. Similar to the proof of the previous lemma, it suffices to construct proper upper and lower solutions. When $c=$ $2 \sqrt{d_{2} r_{2}}$, let

$$
\gamma_{3}=\frac{c+\sqrt{c^{2}-4 d_{1} r_{1}}}{2 d_{1}} .
$$

Fix $\eta>1$ such that

$$
\eta \in\left(1, \min \left\{2, \frac{\gamma_{3}}{\gamma_{1}}, \frac{\gamma_{1}+\gamma_{2} / 2}{\gamma_{1}}\right\}\right) .
$$

Select $N_{1}>1$ such that

$$
\sup _{\xi<-1}\left\{(-\xi+N \sqrt{-\xi}) e^{\gamma_{2} \xi}\right\} \geq 1+b_{2}, \quad N>N_{1} .
$$

Let $\xi_{2}<-1$ be the smaller root of $(-\xi+N \sqrt{-\xi}) e^{\gamma_{2} \xi}=1+b_{2}$. Clearly, if $N \rightarrow \infty$, then $\xi_{2} \rightarrow-\infty$.
By these constants, we define

$$
\begin{aligned}
& \bar{\phi}_{1}(\xi)=\min \left\{1, e^{\gamma_{1} \xi}\right\}, \\
& \underline{\phi}_{1}(\xi)=\max \left\{0, e^{\gamma_{1} \xi}-Q e^{\eta \gamma_{1} \xi}\right\}, \\
& \bar{\phi}_{2}(\xi)= \begin{cases}1+b_{2}, & \xi \geq \xi_{2}, \\
(-\xi+N \sqrt{-\xi}) e^{\gamma_{2} \xi}, & \xi<\xi_{2},\end{cases} \\
& \underline{\phi}_{2}(\xi)= \begin{cases}0, & \xi \geq-R^{2}, \\
(-\xi-R \sqrt{-\xi}) e^{\gamma_{2} \xi}, & \xi<-R^{2},\end{cases}
\end{aligned}
$$

where $N, Q$, and $R$ are positive constants satisfying that

(N1) $N>N_{1}$ is large such that $-d_{2} / 4+r_{2} b_{2}(-\xi)^{5 / 2} e^{\gamma_{1} \xi} / N+$ $r_{2} b_{2}(-\xi)^{2} e^{\gamma_{1} \xi}<0, \xi<\xi_{2}$;

(Q1) $Q \geq Q_{1}>1$ such that $e^{\gamma_{1} \xi}-Q_{1} e^{\eta \gamma_{1} \xi}>0$ implies $(-\xi+$ $N \sqrt{-\xi}) e^{\gamma_{2} \xi}<e^{\gamma_{2} \xi / 2} ;$

(Q2) $Q \geq\left(r_{1}\left(1+a_{1}\right)+r_{1} b_{1}\right) /-\left(d_{1} \eta^{2} \gamma_{1}^{2}-c \eta \gamma_{1}+r_{1}\right)+Q_{1}$, and

(R0) $R_{0}>1$ is a constant such that $\xi<-R_{0}^{2}<-1$ implies $\underline{\phi}_{2}(\xi) \leq \bar{\phi}_{2}(\xi)$,

(R1) $R \geq R_{1}>R_{0}$ such that $\xi<-R_{1}^{2}$ implies $\bar{\phi}_{2}(\xi)<e^{\gamma_{2}^{\prime} \xi}$, where $\gamma_{2}^{\prime}$ satisfies

$$
\begin{aligned}
2 \gamma_{2}^{\prime}-\gamma_{2}>0, & \\
\gamma_{2}^{\prime}+\gamma_{1}-\gamma_{2}>0, & \\
& \gamma_{2}^{\prime} \in\left(0, \gamma_{2}\right),
\end{aligned}
$$

(R2) $R>R_{2}>R_{1}$ such that $d_{2} / 4 \sqrt{(-\xi)^{3}}-r_{2}(1+$ $\left.a_{2}\right) e^{\left(2 \gamma_{2}^{\prime}-\gamma_{2}\right) \xi} / R_{2}>0, \xi \leq-R_{2}^{2}$.

On the first inequality of (12), if $\bar{\phi}_{1}(\xi)=1<e^{\gamma_{1} \xi}$, then

$$
\begin{array}{r}
1-\bar{\phi}_{1}(\xi)-a_{1} \int_{-\tau}^{0} \underline{\phi}_{1}(\xi+c s) d \eta_{11}(s) \\
-b_{1} \int_{-\tau}^{0} \underline{\phi}_{2}(\xi+c s) d \eta_{12}(s) \leq 0
\end{array}
$$

and the result is clear. If $\bar{\phi}_{1}(\xi)=e^{\gamma_{1} \xi}<1$, then

$$
\begin{aligned}
& 1-\bar{\phi}_{1}(\xi)-a_{1} \int_{-\tau}^{0} \underline{\phi}_{1}(\xi+c s) d \eta_{11}(s) \\
& \quad-b_{1} \int_{-\tau}^{0} \underline{\phi}_{2}(\xi+c s) d \eta_{12}(s) \leq 1, \\
& d_{1} \bar{\phi}_{1}^{\prime \prime}(\xi)-c \bar{\phi}_{1}^{\prime}(\xi)+r_{1} \bar{\phi}_{1}(\xi)=\left(d_{1} \gamma_{1}^{2}-c \gamma_{1}+r_{1}\right) e^{\gamma_{1} \xi} \\
& \quad=0
\end{aligned}
$$


which completes the verification on $\bar{\phi}_{1}(\xi)$. On the second inequality, it is evident if $\bar{\phi}_{2}(\xi)=1+b_{2}$. When $\bar{\phi}_{2}(\xi)=$ $(-\xi+N \sqrt{-\xi}) e^{\gamma_{2} \xi}<1+b_{2}$, we have

$$
\begin{aligned}
& r_{2} \bar{\phi}_{2}(\xi)\left[1-\bar{\phi}_{2}(\xi)-a_{2} \int_{-\tau}^{0} \phi_{2}(\xi+c s) d \eta_{22}(s)\right. \\
& \left.+b_{2} \int_{-\tau}^{0} \bar{\phi}_{1}(\xi+c s) d \eta_{21}(s)\right] \leq r_{2} \bar{\phi}_{2}(\xi)[1 \\
& \left.+b_{2} \bar{\phi}_{1}(\xi)\right]=r_{2} \bar{\phi}_{2}(\xi)+r_{2} b_{2} \bar{\phi}_{2}(\xi) e^{\gamma_{1} \xi} \\
& \bar{\phi}_{2}^{\prime}(\xi)=\gamma_{2} \bar{\phi}_{2}(\xi)-e^{\gamma_{2} \xi}-\frac{N e^{\gamma_{2} \xi}}{2 \sqrt{-\xi}} \\
& \bar{\phi}_{2}^{\prime \prime}(\xi)=\gamma_{2}^{2} \bar{\phi}_{2}(\xi)-2 \gamma_{2} e^{\gamma_{2} \xi}-\frac{N \gamma_{2} e^{\gamma_{2} \xi}}{\sqrt{-\xi}}-\frac{N e^{\gamma_{2} \xi}}{4(-\xi)^{3 / 2}} .
\end{aligned}
$$

Therefore,

$$
\begin{aligned}
& d_{2} \bar{\phi}_{2}^{\prime \prime}(\xi)-c \bar{\phi}_{2}^{\prime}(\xi)+r_{2} \bar{\phi}_{2}(\xi) \times\left[1-\bar{\phi}_{2}(\xi)\right. \\
& -a_{2} \int_{-\tau}^{0} \phi_{2}(\xi+c s) d \eta_{22}(s) \\
& \left.+b_{2} \int_{-\tau}^{0} \bar{\phi}_{1}(\xi+c s) d \eta_{21}(s)\right] \leq d_{2}\left[\gamma_{2}^{2} \bar{\phi}_{2}(\xi)\right. \\
& \left.-2 \gamma_{2} e^{\gamma_{2} \xi}-\frac{N \gamma_{2} e^{\gamma_{2} \xi}}{\sqrt{-\xi}}-\frac{N e^{\gamma_{2} \xi}}{4(-\xi)^{3 / 2}}\right]-c\left[\gamma_{2} \bar{\phi}_{2}(\xi)\right. \\
& \left.-e^{\gamma_{2} \xi}-\frac{N e^{\gamma_{2} \xi}}{2 \sqrt{-\xi}}\right]+r_{2} \bar{\phi}_{2}(\xi)+r_{2} b_{2} \bar{\phi}_{2}(\xi) e^{\gamma_{1} \xi} \\
& =-\frac{d_{2} N e^{\gamma_{2} \xi}}{4(-\xi)^{3 / 2}}+r_{2} b_{2}(-\xi+N \sqrt{-\xi}) e^{\gamma_{2} \xi} e^{\gamma_{1} \xi} \\
& =e^{\gamma_{2} \xi}\left[-\frac{d_{2} N}{4(-\xi)^{3 / 2}}+r_{2} b_{2}(-\xi+N \sqrt{-\xi}) e^{\gamma_{1} \xi}\right] \\
& =\frac{e^{\gamma_{2} \xi}}{(-\xi)^{3 / 2}}\left[-\frac{d_{2} N}{4}\right. \\
& \left.+r_{2} b_{2}\left((-\xi)^{5 / 2}+N(-\xi)^{2}\right) e^{\gamma_{1} \xi}\right]=\frac{N e^{\gamma_{2} \xi}}{(-\xi)^{3 / 2}}\left[-\frac{d_{2}}{4}\right. \\
& \left.+\frac{r_{2} b_{2}(-\xi)^{5 / 2} e^{\gamma_{1} \xi}}{N}+r_{2} b_{2}(-\xi)^{2} e^{\gamma_{1} \xi}\right] \leq 0
\end{aligned}
$$

by (N1).
On the third inequality, it is clear if $\underline{\phi}_{1}(\xi)=0$. When $e^{\gamma_{1} \xi}-$ $\mathrm{Q} e^{\eta \gamma_{1} \xi}>0,(\mathrm{Q} 1)$ implies

$$
\begin{aligned}
& r_{1} \underline{\phi}_{1}(\xi)\left[-\underline{\phi}_{1}(\xi)-a_{1} \int_{-\tau}^{0} \bar{\phi}_{1}(\xi+c s) d \eta_{11}(s)\right. \\
& \left.-b_{1} \int_{-\tau}^{0} \bar{\phi}_{2}(\xi+c s) d \eta_{12}(s)\right] \geq-r_{1} \underline{\phi}_{1}^{2}(\xi) \\
& -r_{1} a_{1} \bar{\phi}_{1}(\xi) \int_{-\tau}^{0} \bar{\phi}_{1}(\xi+c s) d \eta_{11}(s)-r_{1} b_{1} \bar{\phi}_{1}(\xi) \\
& \cdot \int_{-\tau}^{0} \bar{\phi}_{2}(\xi+c s) d \eta_{12}(s) \geq-r_{1}\left(1+a_{1}\right) \\
& \cdot \bar{\phi}_{1}^{2}(\xi)-r_{1} b_{1} \bar{\phi}_{1}(\xi) \bar{\phi}_{2}(\xi) \geq-r_{1}\left(1+a_{1}\right) e^{2 \gamma_{1} \xi} \\
& -r_{1} b_{1} e^{\left(\gamma_{1}+\gamma_{2} / 2\right) \xi} .
\end{aligned}
$$

Since

$$
\begin{aligned}
d_{1} \underline{\phi}_{1}^{\prime \prime}(\xi)-c \underline{\phi}_{1}^{\prime}(\xi)+r_{1} \underline{\phi}_{1}(\xi) \\
=e^{\gamma_{1} \xi}\left(d_{1} \gamma_{1}^{2}-c \gamma_{1}+r_{1}\right) \\
\quad-Q e^{\eta \gamma_{1} \xi}\left(d_{1} \eta^{2} \gamma_{1}^{2}-c \eta \gamma_{1}+r_{1}\right) \\
=-Q e^{\eta \gamma_{1} \xi}\left(d_{1} \eta^{2} \gamma_{1}^{2}-c \eta \gamma_{1}+r_{1}\right)
\end{aligned}
$$

then the third is true by (Q2).

We now consider the fourth inequality, which is clear if $\underline{\phi}_{2}(\xi)=0$. When $\underline{\phi}_{2}(\xi)>0$, we have

$$
\begin{aligned}
\underline{\phi}_{2}^{\prime}(\xi)= & \gamma_{2} \underline{\phi}_{2}(\xi)-e^{\gamma_{2} \xi}+\frac{R}{2 \sqrt{-\xi}} e^{\gamma_{2} \xi} \\
\underline{\phi}_{2}^{\prime \prime}(\xi)= & \gamma_{2}^{2} \underline{\phi}_{2}(\xi)-2 \gamma_{2} e^{\gamma_{2} \xi}+\frac{R \gamma_{2}}{\sqrt{-\xi}} e^{\gamma_{2} \xi} \\
& +\frac{R}{4 \sqrt{(-\xi)^{3}}} e^{\gamma_{2} \xi}
\end{aligned}
$$

which implies

$$
\begin{aligned}
& d_{2} \underline{\phi}_{2}^{\prime \prime}(\xi)-c \underline{\phi}_{2}^{\prime}(\xi)+r_{2} \underline{\phi}_{2}(\xi) \\
& =\left(d_{2} \gamma_{2}^{2}-c \gamma_{2}+r_{2}\right) \underline{\phi}_{2}(\xi)-\left(2 d_{2} \gamma_{2}-c\right) e^{\gamma_{2} \xi} \\
& \quad+R e^{\gamma_{2} \xi}\left[\frac{d_{2} \gamma_{2}}{\sqrt{-\xi}}-\frac{c}{2 \sqrt{-\xi}}+\frac{d_{2}}{4 \sqrt{(-\xi)^{3}}}\right] \\
& =\frac{R d_{2} e^{\gamma_{2} \xi}}{4 \sqrt{(-\xi)^{3}}} .
\end{aligned}
$$


Moreover, (R0) and (R1) imply that

$$
\begin{aligned}
& r_{2} \underline{\phi}_{2}(\xi)\left[-\underline{\phi}_{2}(\xi)-a_{2} \int_{-\tau}^{0} \bar{\phi}_{2}(\xi+c s) d \eta_{22}(s)\right. \\
& \left.\quad+b_{2} \int_{-\tau}^{0} \underline{\phi}_{1}(\xi+c s) d \eta_{21}(s)\right] \geq-r_{2}\left(1+a_{2}\right) \bar{\phi}_{2}^{2}(\xi) \\
& \quad \geq-r_{2}\left(1+a_{2}\right) e^{2 \gamma_{2}^{\prime} \xi}
\end{aligned}
$$

and so

$$
\begin{aligned}
& \frac{R d_{2} e^{\gamma_{2} \xi}}{4 \sqrt{(-\xi)^{3}}}-r_{2}\left(1+a_{2}\right) e^{2 \gamma_{2}^{\prime} \xi} \\
& \quad=R e^{\gamma_{2} \xi}\left[\frac{d_{2}}{4 \sqrt{(-\xi)^{3}}}-\frac{r_{2}\left(1+a_{2}\right) e^{\left(2 \gamma_{2}^{\prime}-\gamma_{2}\right) \xi}}{R}\right]>0
\end{aligned}
$$

by (R2), which completes the verification and proof.

Lemma 6. Assume that $d_{1} r_{1}=d_{2} r_{2}$. Then (3) of Theorem 1 is true.

Proof. Utilizing the parameters similar to those in Lemmas 4 and 5 , we define

$$
\begin{aligned}
& \bar{\phi}_{1}(\xi)= \begin{cases}(-\xi+K) e^{\gamma_{1} \xi}, & \xi \leq \xi_{1}, \\
1, & \xi \geq \xi_{1},\end{cases} \\
& \underline{\phi}_{1}(\xi)= \begin{cases}(-\xi-L \sqrt{-\xi}) e^{\gamma_{1} \xi}, & \xi \leq-L^{2}, \\
0, & \xi>-L^{2},\end{cases} \\
& \bar{\phi}_{2}(\xi)= \begin{cases}(-\xi+N \sqrt{-\xi}) e^{\gamma_{2} \xi}, & \xi \leq \xi_{2}, \\
1+b_{2}, & \xi \geq \xi_{2},\end{cases} \\
& \underline{\phi}_{2}(\xi)= \begin{cases}(-\xi-R \sqrt{-\xi}) e^{\gamma_{2} \xi}, & \xi \leq-R^{2}, \\
0, & \xi>-R^{2},\end{cases}
\end{aligned}
$$

where $\xi_{1}<0$ and $\xi_{2}<0$ such that $\bar{\phi}_{1}(\xi)$ and $\bar{\phi}_{2}(\xi)$ are continuous. Similar to the proof of Lemmas 4 and 5, we can complete the proof.

Before ending this paper, we make the following remarks on the minimal wave speed.

Remark 7. In Lin [15] and Pan [16], the authors studied the asymptotic spreading of (1) if $\tau=0$, in which one species spreads in the minimal wave speed of traveling wave solutions. However, (1) does not satisfy the comparison principle of classical predator-prey systems in $[15,16]$; there are also some technique problems in estimating the asymptotic spreading of (1), which will be further investigated in our future research.

Remark 8. From Pan [8], we see that a traveling wave solution with large wave speed decays exponentially as $\xi \rightarrow-\infty$.
However, when the minimal wave speed is concerned, it does not decay exponentially as $\xi \rightarrow-\infty$.

\section{Data Availability}

The data used to support the findings of this study are included within the article.

\section{Conflicts of Interest}

The authors declare that they have no conflicts of interest.

\section{Acknowledgments}

Dongfeng Li was supported by the National Key Research and Development Program of China (no. 2016YFC0402502).

\section{References}

[1] M. R. Owen and M. A. Lewis, "How predation can slow, stop or reverse a prey invasion," Bulletin of Mathematical Biology, vol. 63, no. 4, pp. 655-684, 2001.

[2] N. Shigesada and K. Kawasaki, Biological Invasions: Theory and Practice, Oxford University Press, Oxford, UK, 1997.

[3] G. Lin, "Invasion traveling wave solutions of a predator-prey system," Nonlinear Analysis. Theory, Methods \& Applications, vol. 96, pp. 47-58, 2014.

[4] S. Pan, "Invasion speed of a predator-prey system," Applied Mathematics Letters, vol. 74, pp. 46-51, 2017.

[5] S. R. Dunbar, "Travelling wave solutions of diffusive LotkaVolterra equations," Journal of Mathematical Biology, vol. 17, no. 1, pp. 11-32, 1983.

[6] S. R. Dunbar, "Traveling wave solutions of diffusive LotkaVolterra equations: a heteroclinic connection in R4," Transactions of the American Mathematical Society, vol. 286, no. 2, pp. 557-594, 1984.

[7] S. R. Dunbar, "Traveling waves in diffusive predator-prey equations: periodic orbits and point-to-periodic heteroclinic orbits," SIAM Journal on Applied Mathematics, vol. 46, no. 6, pp. 1057-1078, 1986.

[8] S. Pan, "Convergence and traveling wave solutions for a predator-prey system with distributed delays," Mediterranean Journal of Mathematics, vol. 14, no. 3, 15 pages, 2017.

[9] J.-H. Huang and X.-F. Zou, "Travelling wave solutions in delayed reaction diffusion systems with partial monotonicity," Acta Mathematicae Applicatae Sinica, vol. 22, no. 2, pp. 243-256, 2006.

[10] K. Li and X. Li, "Travelling wave solutions in diffusive and competition-cooperation systems with delays," IMA Journal of Applied Mathematics, vol. 74, no. 4, pp. 604-621, 2009.

[11] G. Lin, W.-T. Li, and M. Ma, "Traveling wave solutions in delayed reaction diffusion systems with applications to multi-species models," Discrete and Continuous Dynamical Systems-Series B, vol. 19, no. 2, pp. 393-414, 2010.

[12] G. Lin and S. Ruan, "Traveling wave solutions for delayed reaction-diffusion systems and applications to diffusive LotkaVolterra competition models with distributed delays," Journal of Dynamics and Differential Equations, vol. 26, no. 3, pp. 583-605, 2014. 
[13] S.-C. Fu, "Traveling waves for a diffusive SIR model with delay," Journal of Mathematical Analysis and Applications, vol. 435, no. 1, pp. 20-37, 2016.

[14] G. Lin, "Minimal wave speed of competitive diffusive systems with time delays," Applied Mathematics Letters, vol. 76, pp. 164$169,2018$.

[15] G. Lin, "Spreading speeds of a Lotka-Volterra predator-prey system: the role of the predator," Nonlinear Analysis. Theory, Methods \& Applications, vol. 74, no. 7, pp. 2448-2461, 2011.

[16] S. Pan, "Asymptotic spreading in a Lotka-Volterra predator-prey system," Journal of Mathematical Analysis and Applications, vol. 407, no. 2, pp. 230-236, 2013. 


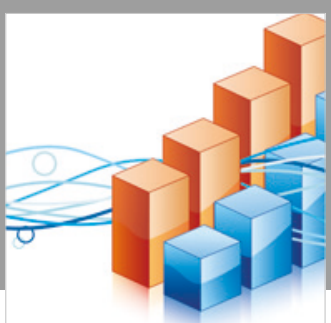

Advances in

Operations Research

\section{-n-m}
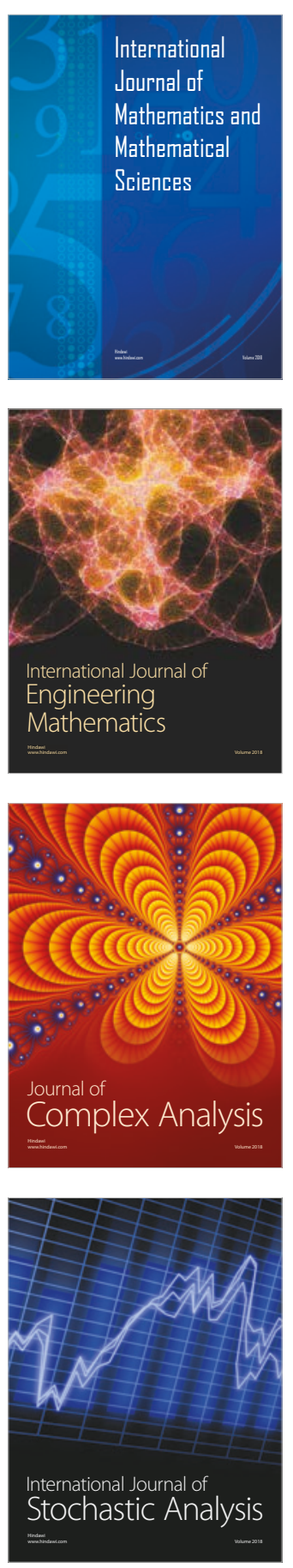
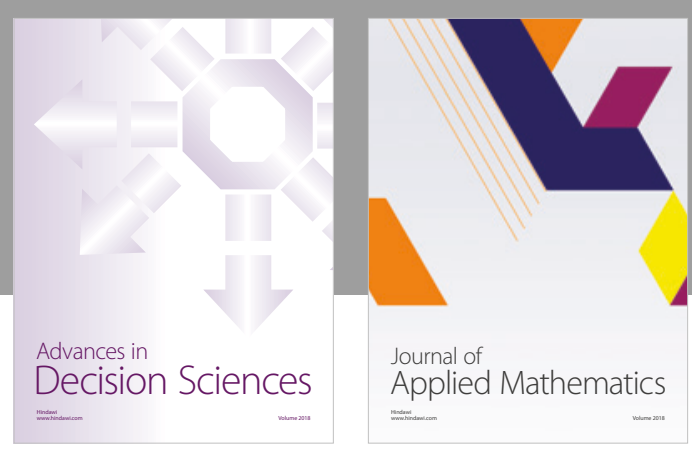

Journal of

Applied Mathematics
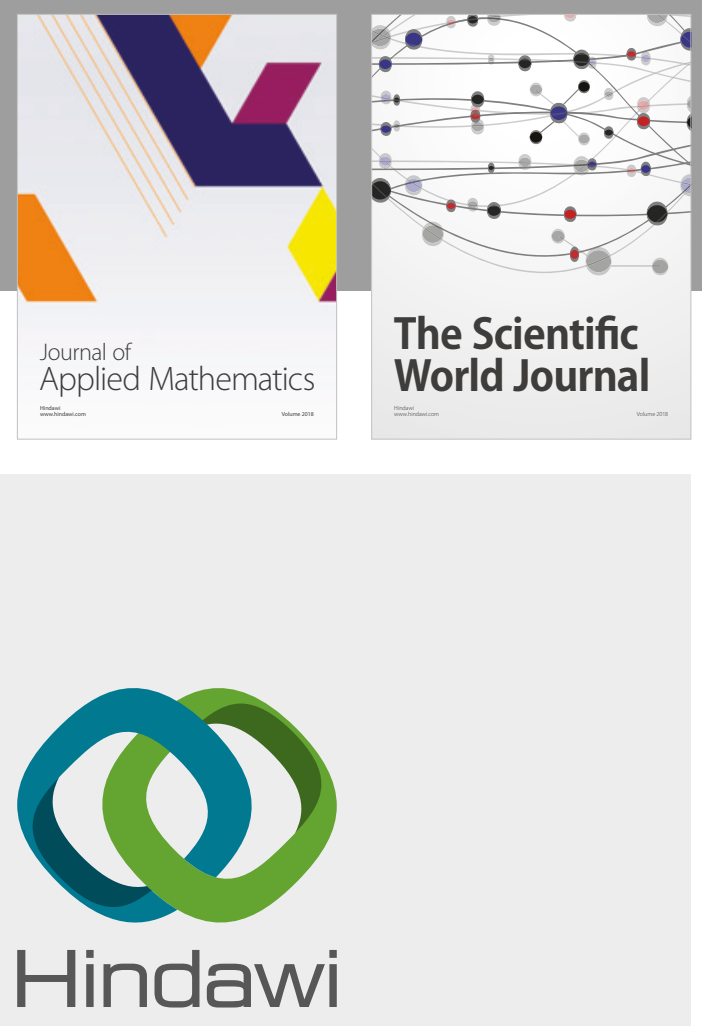

Submit your manuscripts at

www.hindawi.com

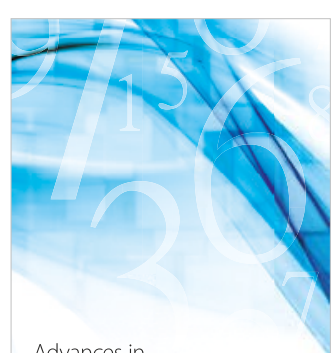

Advances in
Numerical Analysis
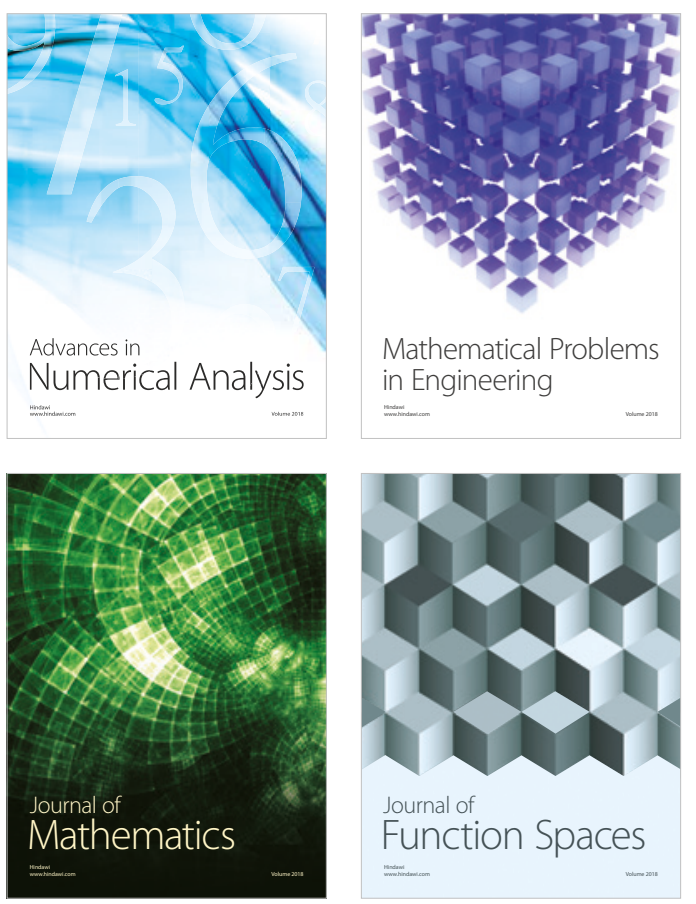

Mathematical Problems in Engineering

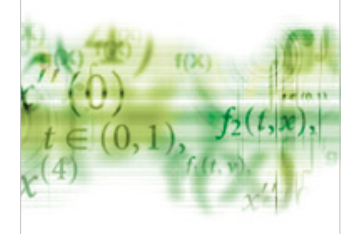

International Journal of

Differential Equations

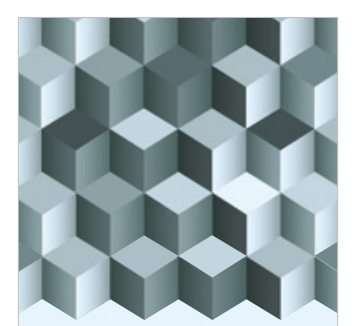

Journal of

Function Spaces

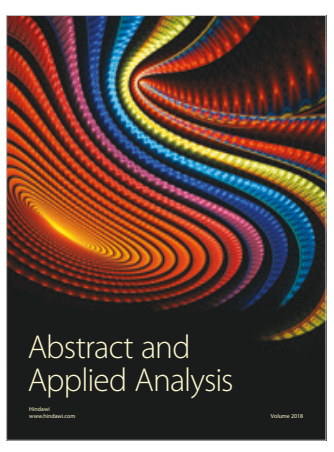

The Scientific

World Journal

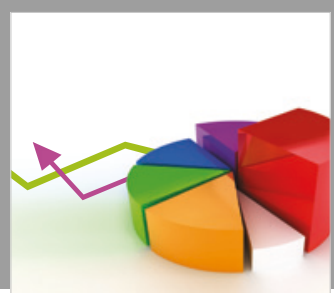

Journal of

Probability and Statistics
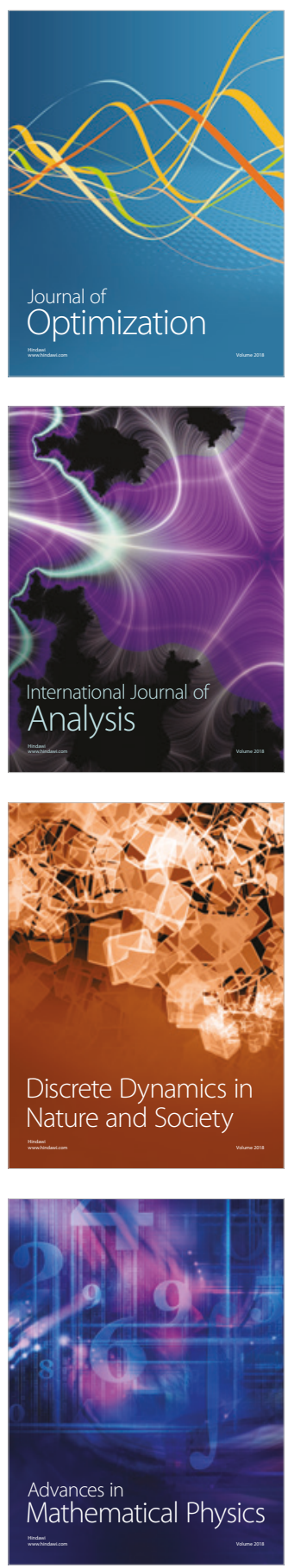